\title{
Optical Properties of Composites of PMMA and Surface-Modified Zincite Nanoparticles
}

\author{
Mustafa M. Demir, Kaloian Koynov, Ümit Akbey, Christoph Bubeck, Insun Park, ${ }^{\dagger}$ \\ Ingo Lieberwirth, and Gerhard Wegner*
}

Max Planck Institute for Polymer Research, Ackermannweg 10, D-55128 Mainz, Germany

Received September 20, 2006; Revised Manuscript Received December 11, 2006

\begin{abstract}
Composites that show visible light transmittance, UV absorption, and moderately high refractive index, based on poly(methyl methacrylate) (PMMA) and zinc oxide (zincite, $\mathrm{ZnO}$ ) nanoparticles, were prepared in two steps. First, surface-modified $\mathrm{ZnO}$ nanoparticles with $22 \mathrm{~nm}$ average diameter were nucleated by controlled precipitation via acid-catalyzed esterification of zinc acetate dihydrate with pentan-1-ol. The surface of growing crystalline particles was modified with tert-butylphosphonic acid $\left(t \mathrm{BuPO}_{3} \mathrm{H}_{2}\right)$ in situ by monolayer coverage. Particle size and graft density of $-\mathrm{PO}_{3} \mathrm{H}_{2}$ on the particle surface were controlled by the amount of surfactant applied to the reaction solution. Second, the surface-modified particles were incorporated into PMMA by in-situ bulk polymerization. Free radical polymerization was carried out in the presence of these particles using AIBN as initiator. Volume fraction $(\phi)$ of the particles was varied from 0.10 to $7.76 \%$ (0.5 to $30 \mathrm{wt} \%)$. Although the particles are homogeneously dispersed in monomer, segregation of the individual particles upon polymerization was observed. Optical constants of the films ca. $2.0 \mu \mathrm{m}$ including absorption and scattering efficiencies, indices of refraction, and dispersion constants were determined. The absorption coefficient at $350 \mathrm{~nm}$ increases linearly with $\mathrm{ZnO}$, obeying Beer's law at low particle contents. However, it levels off toward a value of about $5000 \mathrm{~cm}^{-1}$ and shows a negative deviation at high concentrations because of aggregation of the individual particles. Waveguide propagation loss coefficients of the composite films were examined by prism coupling. A steep increase of the loss coefficient was found with a slope of $52 \mathrm{~dB} \mathrm{~cm}^{-1}$ vol $\%$ as the volume fraction of the particle increases. The refractive index of the composites depends linearly on volume fraction of $\mathrm{ZnO}$ and varies from 1.487 to $1.507(\phi=7.76 \%)$ at $633 \mathrm{~nm}$. The dispersion of refractive index was found to be consistent with Cauchy's formula.
\end{abstract}

\section{Introduction}

The development of polymer-based composites which exhibit various optical functionalities such as high/low refractive index, tailored absorption/emission properties, or strong optical nonlinearities attracts great interest because of the potential optoelectronic applications. ${ }^{1,2}$ More specifically, it was pointed out that such composite materials could be applied as transparent substrate or flexible functional layers of optoelectronic devices which require high transparency in the visible range of the optical spectrum. ${ }^{3}$ Replacing the conventional substrates made up of inorganic glasses by polymer-based materials could provide a number of advantages, as the polymer composites have milder processing conditions and better impact resistance, can be made flexible, and the optical parameters can be tailored. These composites are typically obtained by the incorporation of functional inorganic particles into a transparent polymer matrix. ${ }^{3}$ While the polymeric component provides processability, flexibility, and transparency, the inorganic particles contribute to the desired optical properties. Because of its optical clarity and known chemical and physical properties, poly(methyl methacrylate) (PMMA, Plexiglas) is an excellent host for functional particles. Various types of metal oxide fillers such as $\mathrm{Bi}_{4} \mathrm{Ti}_{3} \mathrm{O}_{12},{ }^{4} \mathrm{SiO}_{2},{ }^{5} \mathrm{TiO}_{2},{ }^{6,7} \mathrm{Nb}_{2} \mathrm{O}_{5},{ }^{8}$ and $\mathrm{Ta}_{2} \mathrm{O}_{5} / \mathrm{SiO}_{2}{ }^{9}$ have been incorporated into polyacrylates to modify the optical properties of these polymers. For example, the refractive indices of PMMA matrix can be increased or decreased by addition of $\mathrm{SiO}_{2}, \mathrm{Al}_{2} \mathrm{O}_{3}$, or $\mathrm{ZrO}_{2}$ filler particles, as Böhm et al. have

\footnotetext{
* Corresponding author: Tel +496131 379 130, Fax +496131 379 330; e-mail wegner@mpip-mainz.mpg.de.

† Present address: Seoul National University, School of Chemistry,
}

indicated. ${ }^{10}$ Lee and Chen ${ }^{7}$ described the preparation of highrefractive-index hybrid films using trialkoxysilane-capped $\mathrm{TiO}_{2}$ particles. The authors reported a linear increase of the refractive index with $\mathrm{TiO}_{2}$ content. Nonlinear optical behavior of the same $\mathrm{TiO}_{2} / \mathrm{PMMA}$ system was investigated by Yuwono et al., ${ }^{6}$ who underlined the nonlinearity of the composites at high filler contents. In addition, binary/mixed metal oxides can be successfully employed giving a wide range of applications. $\mathrm{Ta}_{2} \mathrm{O}_{5^{-}}$ containning $\mathrm{SiO}_{2}$ particles were used in dental polyacrylates because they give enhanced X-ray contrast (radiopacity). ${ }^{9}$ The presence of $\mathrm{SiO}_{2}$ enriches the fraction of inorganic material and thereby increases the tensile strength and hardness of the composite. More importantly, it reduces the shrinkage which inevitably occurs during polymerization. A ferroelectric composite of PMMA with high dielectric constant was obtained by compounding ferroelectric $\mathrm{Bi}_{4} \mathrm{Ti}_{3} \mathrm{O}_{12}$ nanocrystals with the polymer solution. ${ }^{4}$ The optical characterization of the films of these composites was performed by examining interference fringes which are detected in the very thin films on transparent substrates.

The combination of nanosized filler particles with block copolymers and the resulting mesoscopically ordered materials need to be mentioned here as well. It is the interaction of the particles with the self-assembling matrix polymer material which has triggered much interest. ${ }^{11}$ The intriguing feature is that the nanoparticles segregate into only one of the domains which are formed upon solidification of a block copolymer melt, and hierarchically ordered structures are thereby formed. For instance, even within lamellar superstructures the particles tend to migrate to the interface of the domains in which they spontaneously accumulate. This behavior has been experimen- 
tally documented by many authors and has been the subject of theoretical treatment. ${ }^{12-15}$ While segregation of nanosized particles into specific domains of a multiphase system is reasonably understood, it remains still a puzzle what drives such particles to undergo reversible or irreversible association in a homogeneous polymer matrix. Theoretical treatments can be found in the recent literature for instance by Schweizer et al. ${ }^{16}$ and Balazs et al. ${ }^{17}$ However, experimental investigations that would allow to verify theoretical predictions are scarce and, in fact, difficult to obtain for the reason that suitable combinations of nanosized particles of precisely known surface properties and polymer matrices cannot be readily found. In this work we attempt to prepare and modify nanosized zinc oxide $(\mathrm{ZnO})$ with the aim to combine it with PMMA as matrix in order to define a model system.

Zinc oxide $(\mathrm{ZnO})$ is a well-known multifunctional inorganic filler that has unique properties such as strong UV absorption combined with good transparency in the visible range. It is a wide gap semiconductor which shows intense and tunable photoluminescence. The blending of $\mathrm{ZnO}$ nanoparticles with PMMA is believed to improve the optical properties of the polymer. ${ }^{18}$ However, refractive index mismatch (the difference between refractive index of $\mathrm{ZnO}$ and that of PMMA) may cause strong optical scattering which diminishes the transparency of the composites. The mismatch is tolerable when the particle size is much smaller than the wavelength of the incident light (for spherical particles $2 r<\lambda / 10$ ). Moreover, the particles should not be aggregated or clustered into domains. Previous studies have shown that size and size distribution of inorganic domains embedded into a polymer matrix have critical importance for transparency. ${ }^{3,19}$ A population of particles having an average diameter below $50 \mathrm{~nm}$ and a very narrow size distribution is strictly required because scattering intensity is proportional to $r^{3}$ ( $r=$ particle radius). The presence of even a small percentage of particles or aggregates thereof larger than $100 \mathrm{~nm}$ leads to strong scattering in the visible and therefore causes haze or even turbidity. Unfortunately, the formation of strongly associated large aggregates of individual inorganic particles is very likely to occur because of their higher specific surface energy as compared to that of the organic host matrix. ${ }^{19}$ Therefore, the synthesis of nonaggregated particles which can be redispersed in organic media is a major issue in the production of transparent or at least translucent composites.

Different synthetic strategies have been proposed to avoid agglomeration of particles. ${ }^{20}$ There are two principally different routes to incorporate inorganic particles into organics. One method is the in-situ precipitation of particles in the organic phase which may consist of a bulk polymer, polymer solution, or monomer. ${ }^{21-23}$ The rationale is that the isolation and handling of nanoparticles frequently causes irreversible aggregation, and therefore, the particles are nucleated and grown inside the host matrix. It is believed that polymers as hosts do not provide a sufficiently fluid environment to allow individual particles to meet each other by diffusion; thereby, aggregation of the particles may be prevented for reasons of kinetics. The use of (block) copolymers as matrix has been shown to afford opportunities for controlling the formation of the inorganic particles. The copolymer molecules were used as structuredirecting agents developing the domains by microphase separation in which the nanoparticles are formed. ${ }^{22,24-26} \mathrm{Sol}-$ gel processes $^{7,27,28}$ and emulsion precipitation ${ }^{29,30}$ can be involved in this type of approach. However, the drawback is that the polymer may stay contaminated by unreacted educts or byproducts of the precipitation reaction. The second method involves the blending of preformed inorganic particles into the organic medium. ${ }^{9,31,32}$ In an ex-situ synthesis, the particles are prepared separately, isolated, and purified. Subsequently, they are dispersed into the monomer solution or bulk polymer to form composites (blending). Polymerization is carried out in the presence of these particles (in-situ polymerization). In contrast to the former route, the ex-situ synthesis is more compatible with the needs of an industrial production. In the case of PMMA as host matrix it comes as an advantage that this polymer is routinely produced by temperature-induced free radical bulk polymerization on a large scale. From the point of scaling up, dispersing the nanoparticles in the monomer and carrying out its polymerization in the presence of these particles are quite promising. The challenge of this approach is to be able to synthesize nanoparticles in large enough amounts which exhibit good dispersibility in the monomer, have long-term stability against aggregation, and do not negatively interfere with the polymerization process.

Regardless of the type of material and particle size, nanoscale particles tend to aggregate unless they are modified at their surface to decrease the interparticle attraction. The preparation of nanoparticles stabilized by a surface layer of material which acts as surfactant can be accomplished by controlled chemical precipitation. The particles are nucleated in a homogeneous solution and are capped subsequently by surfactant molecules in situ, i.e., in the medium where the particle nucleation has taken place. The chemi- or physisorption of surfactant molecules onto the particle surface terminates the growth of the crystalline particles and modifies their surface properties in the desired direction. This surface modification decreases the surface free energy of the particles and thereby reduces the tendency of agglomeration and their spontaneous growth by Ostwald ripenning. The modified particles can be readily redispersed after isolation in a medium which is compatible with the surfactant layer grafted or deposited to the particle surface.

The selection of a proper surfactant is of utmost importance as they adsorb to surfaces in a substrate specific manner. As an example, thiols are excellent stabilizer for gold, silver, and chalgogenides owing to the strong metal-sulfur bond; Pt and Pd are capped with amines or alkyl cynates; metal oxides are stabilized by acidic surfactants. ${ }^{33}$ In our particular case, although there has been great deal of recent work on $\mathrm{ZnO}$ colloids, the literature lacks information on stabilization of $\mathrm{ZnO}$ nanoparticles in a hydrophobic medium. In this work we report the preparation of $\mathrm{ZnO}$ ("zincite") nanoparticles surface-modified in situ by $t \mathrm{BuPO}_{3} \mathrm{H}_{2}$ using controlled precipitation conditions. In-situ polymerization is carried out giving PMMA/ZnO nanocomposites. Optical characterization of the nanocomposites with regard to absorption and scattering, index of refraction, and its dispersion depending on particle content is reported. Particle segregation is observed and discussed in terms of available theory on the behavior of athermal polymer-particle mixtures. ${ }^{16}$

\section{Experimental Section}

Materials. Zinc acetate dihydrate $\left(\mathrm{Zn}(\mathrm{AcO})_{2} \cdot 2 \mathrm{H}_{2} \mathrm{O}\right)$ (ACS reagent), pentan-1-ol (99\%), 2-methoxyethyl ether (99\%), and tertbutylphosphonic acid $\left(t \mathrm{BuPO}_{3} \mathrm{H}_{2}\right)(98 \%)$ were provided by ACROS and used as received without purification. Tetrabutylammonium acetate (TBAc) $(>90 \%)$ was purchuased from Fluka. $p$-Toluenesulfonic acid monohydrate ( $p$-TSA) (99\%) was obtained from Merck. Methyl methacrylate (MMA) (Acros, stabilized with 10$20 \mathrm{ppm}$ hydroquinone monomethyl ether) was distilled under reduced pressure. 2,2'-Azobis(isobutyronitrile) (Fluka) was recrystallized from methanol. Toluene (ACS grade) was obtained from Riedel de Häen. 
Nanoparticle Synthesis and Surface Modification. Nanoscale zincite particles were precipitated from $\mathrm{Zn}(\mathrm{AcO})_{2} \cdot 2 \mathrm{H}_{2} \mathrm{O}$ in a mixture of pentan-1-ol and 2-methoxyethyl ether. The standard recipe of precipitation is as follows: $0.6 \mathrm{mmol}$ of $p$-TSA, an esterification catalyst, was dissolved in the mixture of pentan-1-ol $(8.5 \mathrm{~mL})$ and 2-methoxyethyl ether $(8.5 \mathrm{~mL})$. The temperature of the solution was fixed to $130{ }^{\circ} \mathrm{C}$, and $2.3 \mathrm{mmol}$ of zinc acetate dihydrate which was grinded beforehand for quick dissolution was added to the solution under magnetic stirring. Reflux was maintained for $1 \mathrm{~h}$. At 30 min of the reaction time, a mixture of $t \mathrm{BuPO}_{3} \mathrm{H}_{2}$ and TBAc $(1: 2 \mathrm{~mol})$ dissolved in an equivolume mixture of pentan-1-ol and 2-methoxyethyl ether ( $3 \mathrm{~mL})$ was added slowly. The coverage of the particle surface by the surfactant was controlled by the amount of $t \mathrm{BuPO}_{3} \mathrm{H}_{2}$ that was added with respect to $\mathrm{Zn}(\mathrm{OAc})_{2} \cdot 2 \mathrm{H}_{2} \mathrm{O}$, and their ratio is designated as $(\mathrm{Zn} / \mathrm{P})$ in the further text. Three different ratios $(\mathrm{Zn} / \mathrm{P})$ were investigated, namely $0.05,0.23$, and 0.34 . The reaction was stopped by quenching the solution to room temperature. The surface-modified particles were isolated by centrifugation at $4000 \mathrm{rpm}$ and washed with ethanol to remove unreacted material. The particles were dried in vacuo at $50{ }^{\circ} \mathrm{C}$ overnight. Yield of the particles was $78 \%$ in the absence of surfactants. It decreases systematically down to $27 \%(\mathrm{Zn} / \mathrm{P}=0.34)$ with increasing $t \mathrm{BuPO}_{3} \mathrm{H}_{2}+\mathrm{TBAc}$ concentration.

Preparation of PMMA/ZnO Nanocomposite Films. Surfacemodified $\mathrm{ZnO}$ nanoparticles were dispersed into MMA, and polymerization was carried out in the presence of these particles at $60{ }^{\circ} \mathrm{C}$ using AIBN as initiator (1.5 wt \%). The volume fraction $(\phi)$ of $\mathrm{ZnO}$ was varied from 0.10 to $7.76 \%(0.5-30$ wt $\%)$. The dispersions in MMA were sonicated for 15 min and kept overnight in the dispersed state to achieve complete wetting of the particle surface. After a second sonication for $30 \mathrm{~min}$, AIBN (1.5 wt \%) was added, and three cycles of a freeze-thaw process were applied prior to polymerization. The glass tubes containing the MMA/ particle mixture were placed into a preheated bath at $60{ }^{\circ} \mathrm{C}$; the polymerization was performed under an argon atmosphere, and it was stopped after the desired time by quenching to room temperature. After polymerization, the bulk nanocomposites were broken into small pieces for dissolution. The polymer/particle composites were dissolved in toluene at room temperature. Composite films were prepared from toluene solution by spin-coating (Headway Research, Inc.) on glass or quartz substrates. The film thickness was controlled by the variation of the solid content of the toluene solution and by the spinning speed. Film thicknesses were measured with a Tencor model P10 step profiler.

Characterization. X-ray diffractograms were obtained from a Seifert XRD 3000 TT diffracometer using $\mathrm{Cu} \mathrm{K} \alpha$ radiation $(\lambda=$ $1.5418 \AA$ ). The size of the particles was determined by dynamic light scattering (DLS) using a Malvern Zetasizer 3000. Thermogravimetry (TGA) (STDA 851 Mettler Toledo) was carried out with a heating/cooling rate of $10 \mathrm{~K} \mathrm{~min}^{-1}$ under nitrogen. Transmission electron microscopy (TEM) was performed with Zeiss 912 Omega working at a voltage of $120 \mathrm{kV}$ and Technai F20 at a voltage of $200 \mathrm{kV}$ SFEG. ${ }^{31} \mathrm{P}$ solid-state NMR spectra were obtained with a Bruker ASX500 spectrometer equipped with a $2.5 \mathrm{~mm}$ fast MAS probe. The refractive indices of waveguides prepared from PMMA$\mathrm{ZnO}$ with typical thicknesses around $1.5-2 \mu \mathrm{m}$ were determined by prism coupling using the $m$-line technique, as described elsewhere. ${ }^{34}$ For these measurements, a symmetric $60^{\circ}$ prism (F2 glass, Schott) was employed and the following lasers: $\mathrm{Kr}$ ion (413 $\mathrm{nm}), \mathrm{Ar}$ ion $(488 \mathrm{~nm})$, and $\mathrm{HeNe}(543,632.8 \mathrm{~nm})$. Both $\mathrm{TE}_{0}$ and $\mathrm{TE}_{1}$ modes could be observed at all wavelengths which allowed to calculate the refractive index and film thickness simultaneously. This procedure ensures an accuracy of the refractive index measurement of about \pm 0.0001 . Furthermore, the obtained values for the films thicknesses were in good agreement with those measured with the step profiler. The Abbe numbers of the prepared composite films were calculated according to ${ }^{35}$

$$
v=\frac{n_{543}-1}{n_{413}-n_{633}}
$$

where $n_{\lambda}$ is the refractive index at $\lambda \mathrm{nm}$. Waveguide loss experiments were performed by use of a $\mathrm{HeNe}(632.8 \mathrm{~nm})$ laser in a setup described earlier. ${ }^{36}$ In short, a propagation mode was excited in the waveguide by prism coupling with a half cut prism (LaSF18 glass, Schott). The scattered light of the waveguide mode was imaged on a diode array. Attenuation loss coefficients $\alpha_{\mathrm{gw}}$ were determined from the scattered light intensity as a function of distance from the coupling prism.

\section{Results and Discussion}

Synthesis of Surface-Modified Nanoparticles. ZnO nanoparticles were nucleated and grown by means of transesterification of $\mathrm{Zn}(\mathrm{OAc})_{2} \cdot 2 \mathrm{H}_{2} \mathrm{O}$ with pentan-1-ol at $130{ }^{\circ} \mathrm{C}$ using 2-methoxyethyl ether as diluent. Particle formation was promoted by a homogeneous esterification catalyst, $p$-toluenesulfonic acid monohydrate ( $p$-TSA). The reaction time required to bring the precipitation to completion is shortened under the given conditions from 2 to $1 \mathrm{~h}$. The catalyst not only accelerates the reaction but also yields particles of monodisperse size distribution. Furthermore, the particles exhibit better crystallinity, as described in our previous work. ${ }^{37}$ For surface modification of the as-synthesized particles, a mixture of $t \mathrm{BuPO}_{3} \mathrm{H}_{2}$ and TBAc was added to reaction mixture while the reaction was taking place. It needs mentioning that addition of the surfactant ("capping agent") at the beginning of the reaction results in the formation of an undesirable zinc phosphonate phase. Therefore, timing of the surfactant addition at $30 \mathrm{~min}$ after the reaction had started is very important. At this time, the nucleation of $\mathrm{ZnO}$ crystals is completed. After the application of the surfactant, the reaction was continued for further $30 \mathrm{~min}$ to achieve a uniform surface coverage. $t \mathrm{BuPO}_{3} \mathrm{H}_{2}$ molecules adsorb onto the surface of growing $\mathrm{ZnO}$ nanocrystals by the coordination of the $-\mathrm{PO}_{3} \mathrm{H}_{2}$ groups to the surface zinc atoms.

The controlled precipitation has two outcomes: (i) the surface layer prevents the diffusion of reactive species to/from the crystal surface and therefore suppresses the crystal growth by Ostwald ripening; (ii) the adsorption modifies the surface properties of the particles and makes them redispersible in a hydrophobic monomer and eventually compatible with the polymer matrix. As a result, hydrophobic nanoscale particles of narrow size distribution, as required for transparency, are obtained.

TBAc is used together with $t \mathrm{BuPO}_{3} \mathrm{H}_{2}$ as an acid conjugate. It plays a key role in the mineralization to ensure that the precipitate appears in the desired $\mathrm{ZnO}$ phase. Figure 1 shows the X-ray diffraction (XRD) patterns of the products prepared with $t \mathrm{BuPO}_{3} \mathrm{H}_{2}$ alone and with the stochiometric mixture of $t \mathrm{BuPO}_{3} \mathrm{H}_{2}+$ TBAc under the same reaction conditions. The product contains the mixture of two phases in the first case: $\mathrm{ZnO}$ and lamellar zinc butylphosphonate. The corresponding XRD pattern in Figure 1a exhibits three symmetric and equidistant reflections at $2 \Theta<20^{\circ}$ while the well-known reflections of crystalline $\mathrm{ZnO}$ appear first at $31.36^{\circ}$. The pattern at $2 \Theta<20^{\circ}$ has features typical for lamellar compounds having a turbostratic structure of poorly ordered character. ${ }^{38}$ In this phase, zinc cations form positively charged layers, and chargecompensating counteranions, $t \mathrm{BuPO}_{3} \mathrm{H}^{-}$and $t \mathrm{BuPO}_{3}{ }^{2-}$, are loosely held in the interlayer region. The first Bragg reflection in the pattern is identified as the $00 l$ interlayer distance $(12.6$ $\AA$ ) which depends on the layer composition. The surfactant that is employed with the intention of surface stabilization of the $\mathrm{ZnO}$ particle core surprisingly enters into mineralization and leads to formation of the lamellar structure as side product. Further, anionic surfactants such as octylphosphonic acid and statistical copolymers of acrylic acid and octadecyl acrylate- 


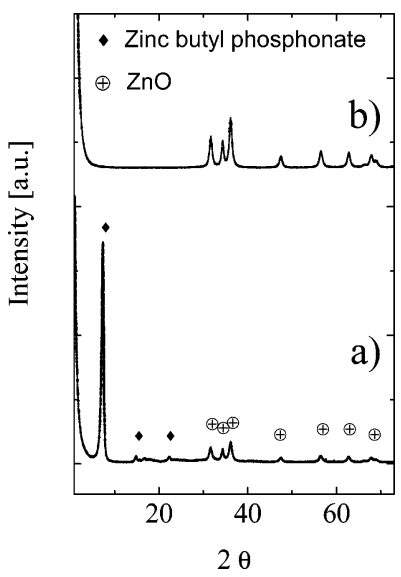

Figure 1. $\mathrm{XRD}$ patterns of the $\mathrm{ZnO}$ powders precipitated in the presence of (a) $t \mathrm{BuPO}_{3} \mathrm{H}_{2}$ and (b) $t \mathrm{BuPO}_{3} \mathrm{H}_{2}+$ TBAc at $130{ }^{\circ} \mathrm{C}$ in $1 \mathrm{~h}$. In the former case, the mixture of $\mathrm{ZnO}$ and undesirable layered structure of zinc butylphosphonate are formed, whereas in the latter the formation of layered structure is suppressed and desired crystal structure of hexagonal $\mathrm{ZnO}$ (JCPDS card no. 36-1451) is obtained.

methacrylic acid also induce the formation of similar intercalation structures with different interlayer distances depending on the length of the alkyl chain.

However, $\mathrm{ZnO}$ (free of side product) was obtained when the mixture of $t \mathrm{BuPO}_{3} \mathrm{H}_{2}+$ TBAc was used (Figure 1b). Tetrabutylammonium ion competes with zinc cation and suppresses the nucleation of the layered structure. It is to be noted that TBAc does not act as a surfactant but is an additive or modifier. It drives the reaction toward formation of $\mathrm{ZnO}$ without having any affinity to the particle surface. Employing TBAc alone (without $t \mathrm{BuPO}_{3} \mathrm{H}_{2}$ ) does not hydrophobize the particles surface even though $\mathrm{ZnO}$ is the only phase obtained. In fact, considering the highly acidic reaction medium due to the presence of $p$-TSA and the high isoelectric point of $\mathrm{ZnO}$ (10.3), ${ }^{39}$ the surface charge of the $\mathrm{ZnO}$ particles is supposedly positive. The association of a cation, e.g., tetrabutylammonium ion, to the positively charged particle surface does not seem plausible. Besides, neither elemental analysis nor vibrational spectroscopy gives an indication of TBAc present on the surface of the $\mathrm{ZnO}$ particles.

Characterization of Zincite Nanoparticles. Grafting of $t \mathrm{BuPO}_{3} \mathrm{H}_{2}$ onto the particle surface was confirmed by both vibrational and NMR spectroscopy. DRIFT spectra (not shown) prove the adsorption of $-\mathrm{PO}_{3} \mathrm{H}_{2}$ to $\mathrm{ZnO}$ surface as similar to the report of Cozzoli et al. ${ }^{40}$ From the DRIFT spectra alone, one can only infer that adsorption has occurred, and conclusions about the exact situation of coordination on the particle surface cannot be drawn. However, the nature of bonding could be elucidated by ${ }^{31} \mathrm{P}$ MAS NMR spectroscopy. The spectra of bulk $t \mathrm{BuPO}_{3} \mathrm{H}_{2}$ and of surface-bound $t \mathrm{BuPO}_{3} \mathrm{H}_{2}\left(t \mathrm{BuPO}_{3} \mathrm{H}_{2} / \mathrm{ZnO}\right)$ are displayed in Figure 2. $t \mathrm{BuPO}_{3} \mathrm{H}_{2}$ is a crystalline solid ${ }^{41}$ that has three chemically inequivalent phosphorus atoms giving three sharp signals at around 41, 42, and $43 \mathrm{ppm}$. The spectrum of $t \mathrm{BuPO}_{3} \mathrm{H}_{2} / \mathrm{ZnO}$ is similar to those reported for different metal oxide powders modified with phosphonic acid derivatives. ${ }^{42-45}$ The resonances are considerably broadened as compared to those of unbound acid, namely from 575 to $4711 \mathrm{~Hz}$. The large line width and chemical shifts arise from a distribution of binding sites and modes of the phosphonic acid group on the heterogeneous $\mathrm{ZnO}$ surface. The peaks are attributed to phosphonic acid bound to the particles by one, two, and three $\mathrm{Zn}-\mathrm{O}-\mathrm{P}$ bonds. A comparison of the integral signal intensities suggests that binding occurs via mainly tridentate and bidentate modes. In addition to the zinc phosphonic acid species, the surface of the $\mathrm{ZnO}$ particles contains acetate and hydroxyl groups as well.
Acetate originates from the starting material $\left(\mathrm{Zn}(\mathrm{OAc})_{2} \cdot 2 \mathrm{H}_{2} \mathrm{O}\right)$ and exists at the surface as a mixture of unidentate, bidentate, and chelate type of structures. Hydroxyl groups are inevitably present on the surface of metal oxides ${ }^{46}$ unless they are annealed at high temperature in vacuo. Since particle formation is catalyzed by $p$-TSA, the attachment of this species to the crystal surface might also be considered. However, $p$-TSA adsorbs to the particles' surface very loosely if at all and is removed completely by washing of the particles. ${ }^{37}$

The competitive interaction of carboxylic and phosphonic acids with metal oxide surfaces has been known from the studies dealing with structures formed by the adsorption of carboxyalkylphosphonic acids on transition metal oxide powders. ${ }^{42,43,45}$ These studies pointed out that phosphonic acids bind selectively to metal oxides and form densely packed layers covering their surface. Phosphor compounds also bind to metal oxide surfaces more strongly than carbon compounds. ${ }^{44}$ Therefore, the desorption temperatures of alkyl phosphonates are higher than those of corresponding carboxylates. ${ }^{47}$ TGA of the $\mathrm{ZnO}$ particles and the ones modified with $t \mathrm{BuPO}_{3} \mathrm{H}_{2}$ suggest that mass loss takes place at different temperatures. Particles prepared without addition of $t \mathrm{BuPO}_{3} \mathrm{H}_{2}$ show mass loss at $230{ }^{\circ} \mathrm{C}$ due to the desorption of the surface acetate and surface-bound water molecules. On the other hand, phosphonic acid functionalized particles have reproducibly the same desorption temperatures at $429{ }^{\circ} \mathrm{C}$, but the mass loss depends on $\mathrm{P} / \mathrm{Zn}$ which was fixed by the preparation conditions. This is shown in column 6 of Table 1. The normalized weight losses exhibit a systematic increase with the increase of $\mathrm{Zn} / \mathrm{P}$. TGA of the particles prepared without surfactant show $1.24 \%$ normalized loss.

The presence of surface-anchored tert-butylphosphonate and its graft density have a strong influence on the particle size and particle size distributions (PSD) in MMA. Figure 3 presents DLS number size distributions of the neat particles and the particles treated with different amounts of $t \mathrm{BuPO}_{3} \mathrm{H}_{2}$ and TBAc. While the neat particles exhibit micron scale diameters and broad size distribution of most probably aggregated structures, the ones treated with $t \mathrm{BuPO}_{3} \mathrm{H}_{2}$ show diameters of individual particles with a short tail of the size distribution ending at around $80 \mathrm{~nm}$ at $\mathrm{P} / \mathrm{Zn}=0.34$. There is a systematic decrease of mean diameter and narrowing of size distribution with increasing $\mathrm{P} / \mathrm{Zn}$. The graft density of $-\mathrm{PO}_{3} \mathrm{H}_{2}$ can be estimated from the difference in mass loss between the surface-modified and bare particles taking into account their known diameters obtained from the DLS measurements. For $\mathrm{P} / \mathrm{Zn}$ of 0.34 , the value is 2.5 phosphonic groups $/ \mathrm{nm}^{2}$, which corresponds to $\sim 50 \%$ coverage, assuming the cross-sectional size of $\mathrm{PO}_{3}$ moiety as $24 \AA .2,48$

Elemental analysis of the particles was performed selectively for phosphor and zinc by use of an energy dispersive spectrometer (EDS) attached to TEM. The density of phosphor with respect to zinc was figured out and is presented in column 7 of Table 1. Similar to the results obtained by TGA, increasing the surfactant concentration in the preparation increases the surface coverage. A qualitative conclusion which may be drawn from both TGA and EDS results is that the adsorption of $t \mathrm{BuPO}_{3} \mathrm{H}_{2}$ onto the particle surface follows the Langmuir type isotherm. The amount of adsorbed groups levels off toward high concentrations of surfactant, owing to the formation of a compact monolayer on the particle surface.

The disadvantage of increasing the amount of $t \mathrm{BuPO}_{3} \mathrm{H}_{2}+$ TBAc is the gradual loss of the product yield in particle synthesis, as shown in Table 1 . In fact, the combination of $t \mathrm{BuPO}_{3} \mathrm{H}_{2}$ and TBAc yields salts of the phosphonate and acetic acid. Since $\mathrm{ZnO}$ is soluble in acidic media, the more $t \mathrm{BuPO}_{3} \mathrm{H}_{2}$ 

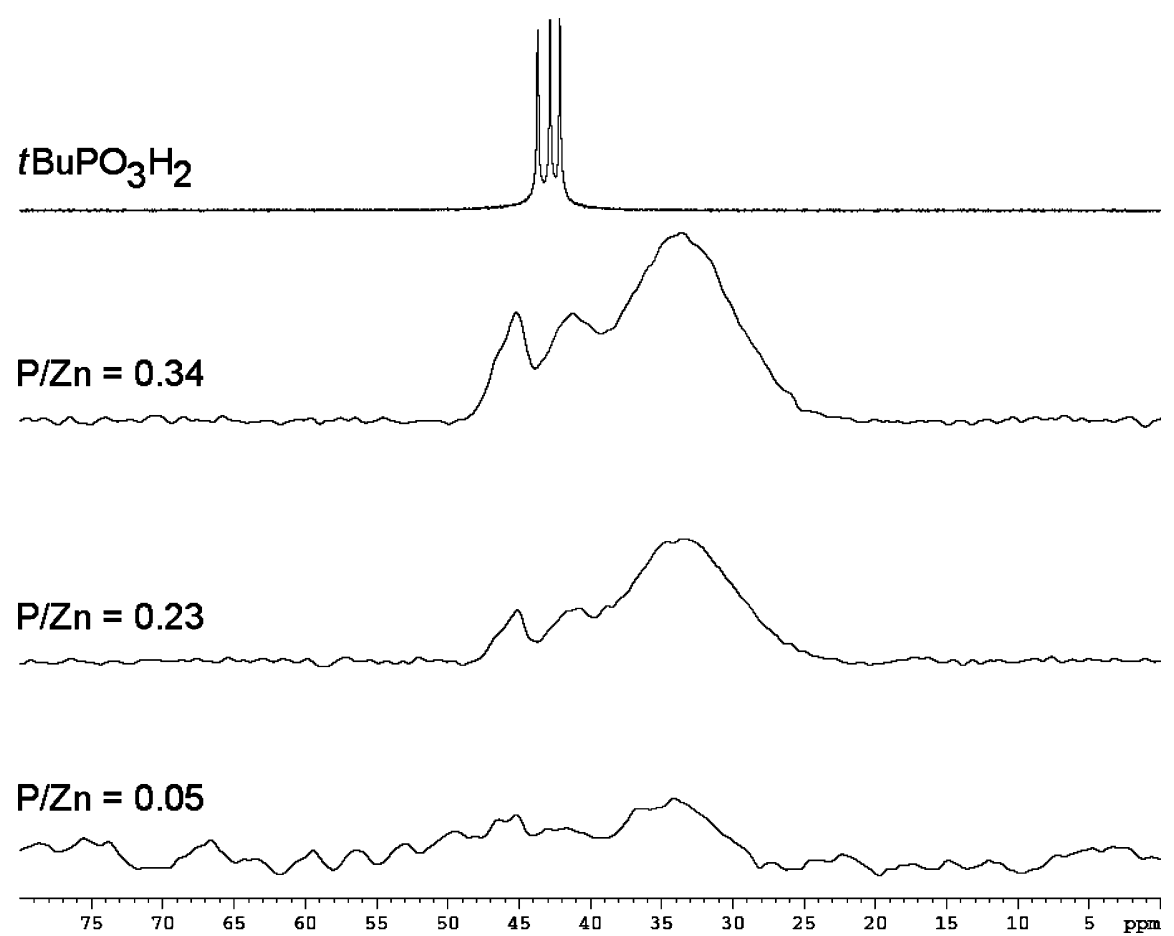

Figure 2. NMR MAS ${ }^{31} \mathrm{P}$ of $t \mathrm{BuPO}_{3} \mathrm{H}_{2}$ and of $t \mathrm{BuPO}_{3} \mathrm{H}_{2}$ modified $\mathrm{ZnO}$ particles (see text for definition of $\mathrm{P} / \mathrm{Zn}$ ).

Table 1. Characterization of $\mathrm{ZnO}$ Nanoparticles with and without Surface Modification by $t \mathrm{BuPO}_{3} \mathrm{H}_{2}$

\begin{tabular}{|c|c|c|c|c|c|c|c|}
\hline $\begin{array}{c}\text { samples } \\
\mathrm{P} / \mathrm{Zn}\end{array}$ & $\begin{array}{c}\text { yield, } \\
\text { gravimetry (\%) }\end{array}$ & $\begin{array}{l}\text { mean diameter of the } \\
\text { particles in MMA }(n m)\end{array}$ & $\begin{array}{l}\text { fwhm of } \\
\operatorname{PSD}^{a}(\mathrm{~nm})\end{array}$ & $\begin{array}{c}\text { crystallite size }{ }^{b} \\
L_{(002)}(\mathrm{nm})\end{array}$ & $\begin{array}{c}\text { TGA mass } \\
\operatorname{loss}^{c}(\%)\end{array}$ & $\begin{array}{l}\text { EDS elemental } \\
\text { analysis }^{d} \mathrm{P} / \mathrm{Zn}\end{array}$ & $\begin{array}{l}\text { surface coverage } \\
\text { by } t \mathrm{BuPO}_{3} \mathrm{H}_{2}(\%)\end{array}$ \\
\hline 0 & 78 & 668 & 483 & 29 & 1.24 & 0 & 0 \\
\hline 0.05 & 69 & 49 & 34 & 17 & 2.04 & 0.015 & 21 \\
\hline 0.23 & 48 & 36 & 25 & 15 & 2.88 & 0.036 & 43 \\
\hline 0.34 & 27 & 22 & 18 & 12 & 3.14 & 0.039 & 50 \\
\hline
\end{tabular}

${ }^{a}$ Full width at half-maximum of particle size distribution obtained from DLS (Figure 3). ${ }^{b}$ Estimated from Scherrer's formula. ${ }^{c}$ Normalized mass loss under helium. ${ }^{d}$ Average percent of $\mathrm{P}$ with respect to $\mathrm{Zn} .{ }^{e}$ Estimated from the mass loss in TGA and assuming the monolayer formation.

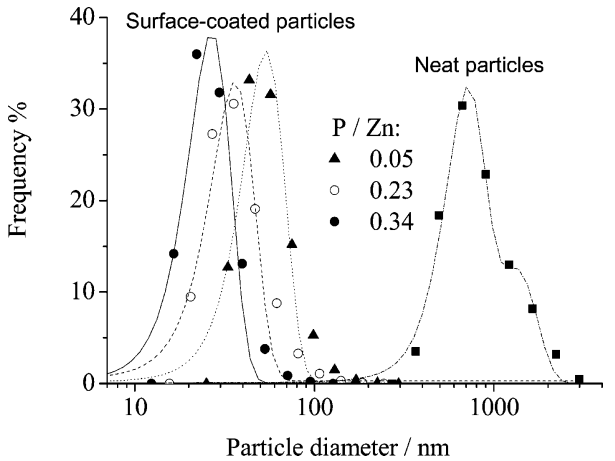

Figure 3. DLS number size distributions of the neat $\mathrm{ZnO}$ particles and the particles modified with different amount of surface-grafted $t \mathrm{BuPO}_{3} \mathrm{H}_{2}$ in MMA as medium.

is added with the aim to increase the graft density the more is the acidity of medium increased which leads to a situation that the surface of the formed particles is etched away. The product yield decreases gradually from $78 \%$ down to $27 \%(\mathrm{P} / \mathrm{Zn}=0.34)$. $\mathrm{P} / \mathrm{Zn}$ was fixed at 0.34 for our further steps of composite preparation because under these conditions the smallest average size of $\mathrm{ZnO}$ was obtained (e.g., Figure 3).

Figure $4 \mathrm{a}$ illustrates the transparency of dispersions of the neat and surface-modified $\mathrm{ZnO}$ particles in MMA $\left(3 \mathrm{mg} \mathrm{mL}^{-1}\right)$. The suspension shown on the right-hand side is opaque because light is scattered by the large aggregated particles. However, the one prepared with the surface-modified particles on the lefthand side is transparent. The undesirable aggregation of primary particles, and accordingly the optical scattering, is avoided by the efficient surface modification. Figure $4 \mathrm{~b}$ is the bright-field TEM micrograph of surface-modified $\mathrm{ZnO}$ particles cast from a dispersion in MMA, showing that the dispersion consisted of mainly separated $\mathrm{ZnO}$ nanocrystallites of a diameter $<25 \mathrm{~nm}$. A rather uniform distribution of the hydrophobic nanoparticles was achieved almost free of aggregation over the whole field of view of more than $40 \mu \mathrm{m}^{2}$. However, a tiny amount of still aggregated material was occasionally observed near the edge of the cupper grid. A high-resolution image of a selected aggregate from this image is presented in Figure 4c. The appearance of lattice fringes indicates the excellent crystallinity of the as-synthesized particles. Crystallite size of the particles was determined from the line broadening of the (002) reflection of the XRD pattern using Scherrer's formula: ${ }^{49}$

$$
L=\frac{\alpha \lambda}{\beta \cos \theta}
$$

where $L$ is the mean particle size, $a$ is a geometric factor equal to $0.94, \lambda$ is the $\mathrm{X}$-ray wavelength $(1.5406 \AA)$, and $\beta$ is the half-width of the diffraction peak. The size of the crystallite particles was found to be $29 \mathrm{~nm}$. A systematic decrease of crystallite size is observed with increasing the amount of surfactant employed (column 5 of Table 1). For example, this value is more than halved to $12 \mathrm{~nm}$ when $\mathrm{P} / \mathrm{Zn}$ was 0.34 .

Preparation of PMMA-Zincite Composite. The polymer/ particle composites were prepared by bulk polymerization of a dispersion of the $\mathrm{ZnO}$ particles in the monomer. The surfacefunctionalized $\mathrm{ZnO}$ particles interfere with the process of free 


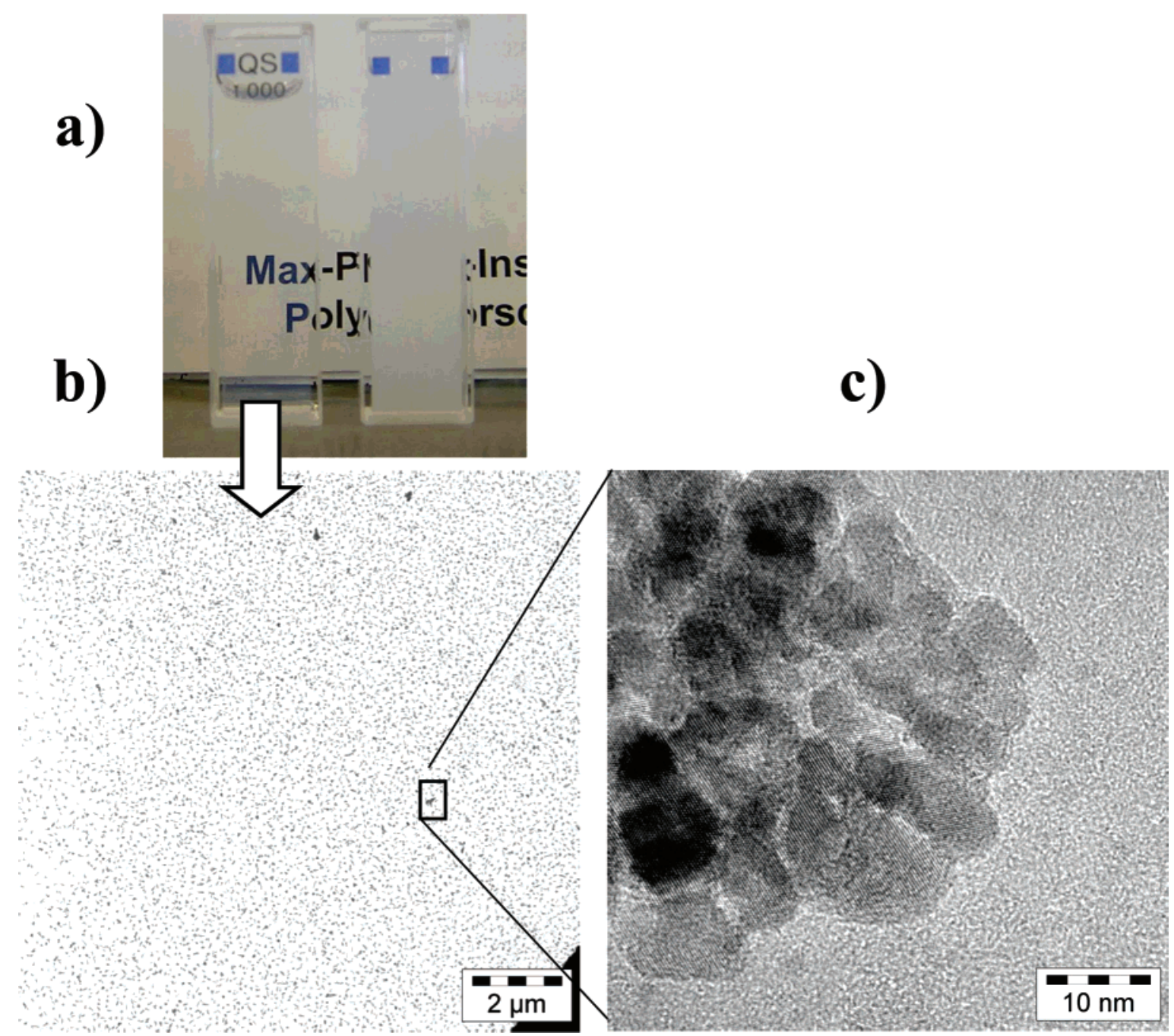

Figure 4. (a) Photographs of the $\mathrm{ZnO} / \mathrm{MMA}$ dispersions prepared with coated (left) and bare (right) particles. The weight fraction of $\mathrm{ZnO}$ particles was $3 \mathrm{mg} \mathrm{mL} \mathrm{m}^{-1}$ for both cases. (b) A drop from the left dispersion was cast on carbon film of a TEM grid. After the evaporation of the dispersing monomer, the surface of the grid is examined by microscopy. (c) High-resolution transmission microscopy image of a particle aggregate from the left image.

radical polymerization of MMA, as described in detail elsewhere. ${ }^{50}$ The surface of the modified $\mathrm{ZnO}$ nanoparticles acts as a transfer agent, thereby regulating both the molecular weight distribution and the nature of end groups of PMMA. However, the bulk polymerization can be carried out to completion, and high molecular weight PMMA is obtained. The molecular weights are on the order of $100 \mathrm{~kg} \mathrm{~mol}^{-1}$. The tacticity of polymer is independent of the presence of particles, and it is described by $20 \%$ of isotactic and $80 \%$ of syndiotactic triads. The glass transition temperature $\left(T_{\mathrm{g}}\right)$ of the composites is independent of the particle content. $T_{\mathrm{g}}$ was found to be positioned between 102 and $109{ }^{\circ} \mathrm{C}$, very close to the $T_{\mathrm{g}}$ of atactic PMMA $\left(105^{\circ} \mathrm{C}\right)$. In other words, there is no specific physical interaction between the particles and the polymer in the bulk, and the system is athermal.

Although the particles are well dispersed in the monomer, the polymerization or, rather, presence of polymer induces the aggregation of the primary particles. ${ }^{50}$ Figure 5 shows TEM images of thin sections of the composites at various content of $\mathrm{ZnO}$ before dissolution of the composites in toluene for spincoating. At low $\mathrm{ZnO}$ content, the domains of inorganic material are aggregates of loosely associated primary particles (see inset of Figure 5a). Up to a volume fraction $(\phi)$ of $1.24 \%$, the aggregates with nearly $100 \mathrm{~nm}$ of diameter consist on average of 4-6 individual particles. These domains are embedded in the PMMA matrix and have no mutual contact. Increasing the particle content causes a fractal type of aggregation, as shown by Figure 5d. Further increase of the $\mathrm{ZnO}$ particle volume fraction makes the domains larger and causes their overlap, as can be seen in Figure $5 d-f$. Percolation of the domains is observed to occur when the sample contains $\phi=2.37 \%$ of nanosized $\mathrm{ZnO}$ particles. The aggregates and individual particles are homogeneously dispersed all over the samples, and they do not form micron-scale inorganic domains (coarse agglomerates).

In fact, the information one can get from the TEM micrographs is restricted to the field of view of the micrograph which is, in reality, a tiny part of the whole specimen. In contrast, optical methods examine the whole population of the inorganic domains over the entire volume of the sample within the probing light beam. In such heterogeneous systems, transmission of the films of composites over the visible region is better suited to follow the aggregation of particles since large domains give rise to transmission loss due to scattering. This will be discussed in the following section.

Optical Properties of the PMMA/Zincite Composites. In order to evaluate the influence of the $\mathrm{ZnO}$ content on the optical transparency of the composites, we studied a series of films in which the volume fraction of $\mathrm{ZnO}$ nanoparticles was varied from 0.10 to $7.76 \mathrm{vol} \%$. The films were prepared by spin-coating of a solution of the bulk polymerized $\mathrm{ZnO} / \mathrm{PMMA}$ composite in 


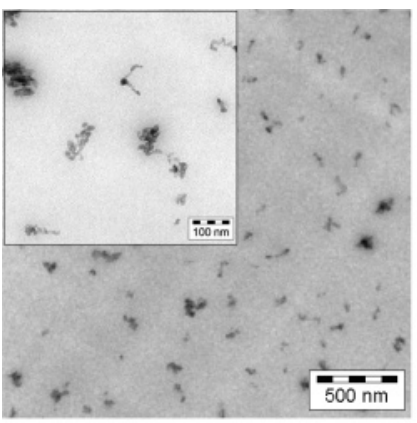

a)

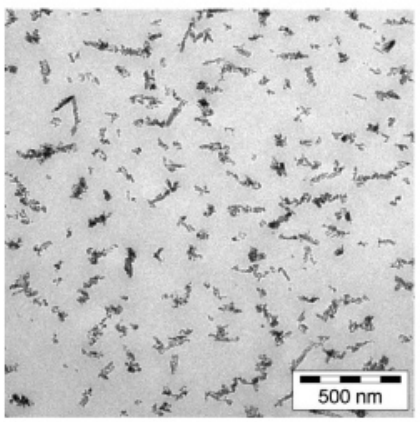

c)

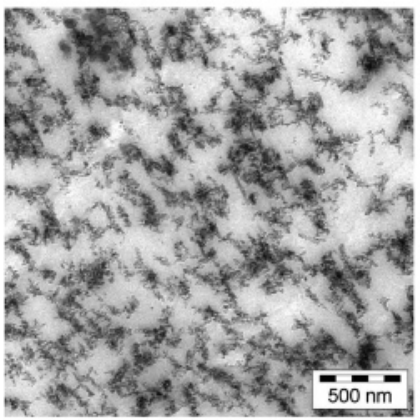

e)

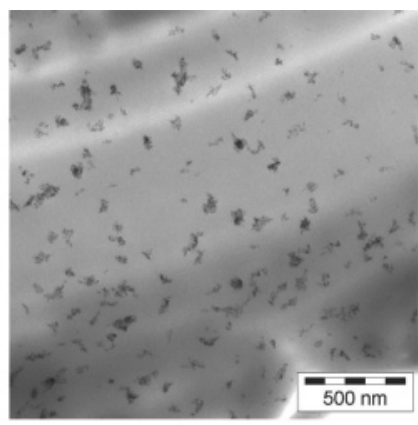

b)

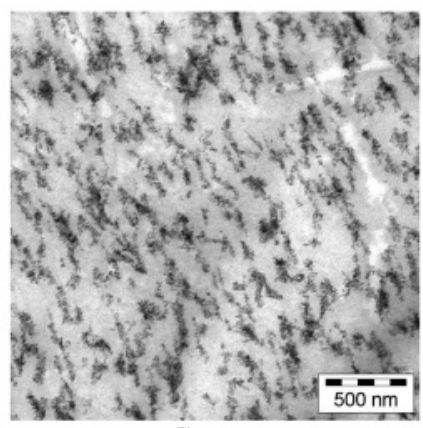

d)

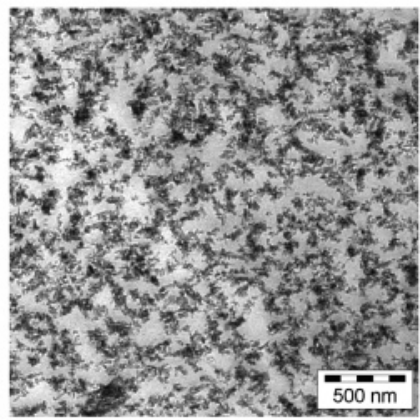

f)
Figure 5. TEM images obtained from thin sections of the bulk composites after polymerization. The percent volume fractions of $t \mathrm{BuPO}_{3} \mathrm{H}_{2}$ modified $\mathrm{ZnO}$ particles are (a) 0.40 , (b) 0.81 , (c) 1.24 , (d) 2.37, (e) 4.68, and (f) 7.76 .

toluene on fused silica substrates. These films had a typical thickness of ca. $2.0 \mu \mathrm{m}$. The transmission UV-vis spectra of these composite films show, not surprisingly, the influence of the $\mathrm{ZnO}$ present in the samples. This is demonstrated by Figure 6a where the optical transmissions $T$ of the films at two different wavelengths in the visible and in the UV, namely at 550 and $350 \mathrm{~nm}$, are plotted as a function of the $\mathrm{ZnO}$ content.

As $\mathrm{ZnO}$ is not absorbing in the visible, only the scattering of the nanoparticles may cause an intensity loss in this spectral region. Indeed, as can be seen from Figure $6 \mathrm{a}$ at $550 \mathrm{~nm}$, the films having $\mathrm{ZnO}$ content up to $\phi=2.37 \%$ exhibit high transmission of approximately $91.3 \pm 0.7 \%$. This value is very close to that of pure PMMA, namely $92 \%$ for normal incidence. In this concentration range, our particles are small enough so that scattering losses are negligible, and the composite films are almost as transparent as PMMA itself. It is important to emphasize here that the transmission of the pure PMMA films for $550 \mathrm{~nm}$ light at normal incidence is about $92 \%$ only as a result of the reflection losses on air/film, film/substrate, and substrate/air interfaces and not because of absorption and scattering losses. For the films having higher $\mathrm{ZnO}$ contents, for example for the one with $7.76 \mathrm{vol} \%$, the intensity decreases to $87.2 \%$, which corresponds to $\sim 5 \%$ intensity loss. At high filler
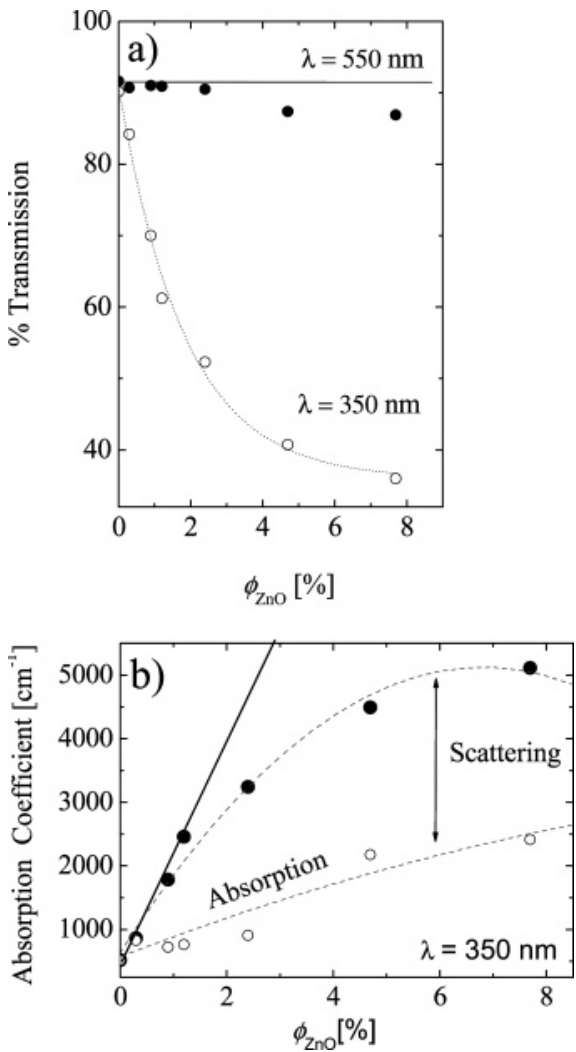

Figure 6. (a) Normal incidence transmission of $2.0 \mu \mathrm{m}$ thick composite films depending on volume fraction of surface-modified $\mathrm{ZnO}$ particles $(\mathrm{Zn} / \mathrm{P}=0.34$; see Table 1$)$ at $550 \mathrm{~nm}$ (full symbols) and $350 \mathrm{~nm}$ (open symbols). The solid line represents the scattering losses at $550 \mathrm{~nm}$, as predicted from eq 3 . The dashed line is a fit to the experimental points with an exponential decay function. (b) Absorption coefficient of PMMA and PMMA-ZnO films at $350 \mathrm{~nm}$ (filled symbols). The solid line is a linear fit to the measured data at low $\mathrm{ZnO}$ volume fractions. The open symbols refer to absorption coefficient after correction for scattering (see text).

contents, particles have higher probability to undergo aggregation $^{51}$ and inevitably cause optical scattering. Transmission loss for such a multiphase system depends on several parameters, namely the size of the inorganic domains, concentration of particles, refractive index difference between the particles and polymer matrix, and thickness of the films studied. The loss originating from scattering can be estimated from the following formula: 52

$$
T=\exp \left[-\frac{3 \phi r^{3}}{4 \lambda^{4}}\left(\frac{n_{\mathrm{p}}}{n_{\mathrm{m}}}-1\right)\right] s
$$

where $T$ is the transmission, $s$ is the optical path length (thickness of the film), $r$ is the particle radius, $\phi$ is the volume fraction of particles, $\lambda$ is the wavelength of the incident light, and $n_{\mathrm{p}}$ and $n_{\mathrm{m}}$ are the refractive indices of the particles and the polymer matrix, respectively. The full line in Figure 6a refers to the prediction of this equation for our PMMA/ZnO composite films where the particle diameter was taken as $2 r=22 \mathrm{~nm}$ (the value obtained from DLS) and the thickness of the films was $2.0 \mu \mathrm{m}$. The values of $n_{\mathrm{p}}$ and $n_{\mathrm{m}}$ are $2.02^{53}$ and $1.49,{ }^{54}$ respectively, at $589 \mathrm{~nm}$. The prediction agrees with the experimental results at low filler contents. However, a deviation appears at higher particle contents when aggregates are formed and start to overlap and eventually percolate through the system. The presented eq 3 is based on the assumption of single scatterers which scatter the light independently. It is certainly reasonable to assume that the particles embedded in the polymer 
matrix are Rayleigh scatterers since their diameter is at least 1 order of magnitude smaller than the wavelength of the incident light beam. However, these assumptions hold only for very dilute systems. In fact, depending on the density of scattering centers, the radiation may be scattered many times which is known as multiple scattering. Increasing the particle content not only increases the number of scattering centers but also enlarges the size of particles because of aggregation as shown by TEM micrographs. Multiple scattering by the pseudoparticles (aggregates or domains) larger than Rayleigh scatterers (in Mie scattering regime) appears at filler contents $\phi \geq 2 \%$ and leads to deviation from the simple prediction.

$\mathrm{ZnO}$ is a wide band gap semiconductor, and it has a high absorption coefficient in the UV. ${ }^{55}$ That is why, in contrast to the visible, the composite films are strongly absorbing in the UV region. As indicated in Figure 6a, the transmission at 350 $\mathrm{nm}$ exhibits an exponential decay (dotted line to the data points) with the particle content. Figure $6 \mathrm{~b}$ presents the absorbance at $350 \mathrm{~nm}$ of the thin composite films (full symbols) at different content of the same $\mathrm{ZnO}$ particles, calculated from the measured transmissions $T$ after correction for the reflection losses on air/ film, film/substrate, and substrate/air interfaces. ${ }^{56}$ It can be seen that absorption coefficient at $350 \mathrm{~nm}$ increases initially linearly with the $\mathrm{ZnO}$ content. As Beer's law predicts, absorbance (absorption + scattering) is directly proportional to the concentration of the absorbing species. However, a negative deviation is observed at $\mathrm{ZnO}$ contents $\phi \geq 2.0 \%$ when aggregation of the nanoparticles becomes significant. In fact, the law holds for homogeneous systems; i.e. for the case of homogeneous distribution of the individual particles in a matrix. As we can see from the TEM micrographs, particles only exist individually at low concentrations. They segregate and form domains containing a number of individual particles as the concentration increases. The segregation leads to inhomogeneous distribution of particles into domains giving highly absorbing cloudy regions in a PMMA matrix depleted of the particles. Because of the packing of the particles into aggregates, the effective volume occupied by the particles, and accordingly the absorption cross section, decrease relatively as compared to a homogeneous distribution. The absorption coefficient of the spin-coated films levels off toward a value of about $5 \times 10^{3}$ $\mathrm{cm}^{-1}$. However, extrapolation of the initial slope to $100 \% \mathrm{ZnO}$ content gives a value of the absorption coefficient $154 \times 10^{3}$ $\mathrm{cm}^{-1}$, which is consistent with the reported value of bulk $\mathrm{ZnO} .{ }^{57}$

For such a heterogeneous polymer-particle system, absorbance is dominated by scattering over whole optical spectrum. However, in the UV region, attenuation of the incident radiation occurs by both scattering and absorption. The contribution by scattering can be estimated by invoking that the scattering efficiency relates to wavelength as $I_{\mathrm{S}} \approx 1 / \lambda^{4}$ as a first approximation. ${ }^{58}$ Therefore, intensity loss originating from scattering at $350 \mathrm{~nm}$ was calculated knowing the loss of the same sample at $550 \mathrm{~nm}$ where the system is nonabsorbing (Figure 6a). The absorbance was corrected for scattering by subtracting the calculated scattering intensity from the measured transmission (Figure 6b). As a result, one can see that absorption and scattering contribute about equally to the observed absorbance of the system at $350 \mathrm{~nm}$. The fluctuation of the data points at low concentrations is the outcome of interference in the transmission spectra over visible due to the multiple reflections of the composite films from substrate and air interfaces.

At low concentrations the majority of particles are homogeneously dispersed and free of coarse agglomerates as Beer's law requires (Figure $5 \mathrm{a}-\mathrm{c}$ ). The presence of a small amount of
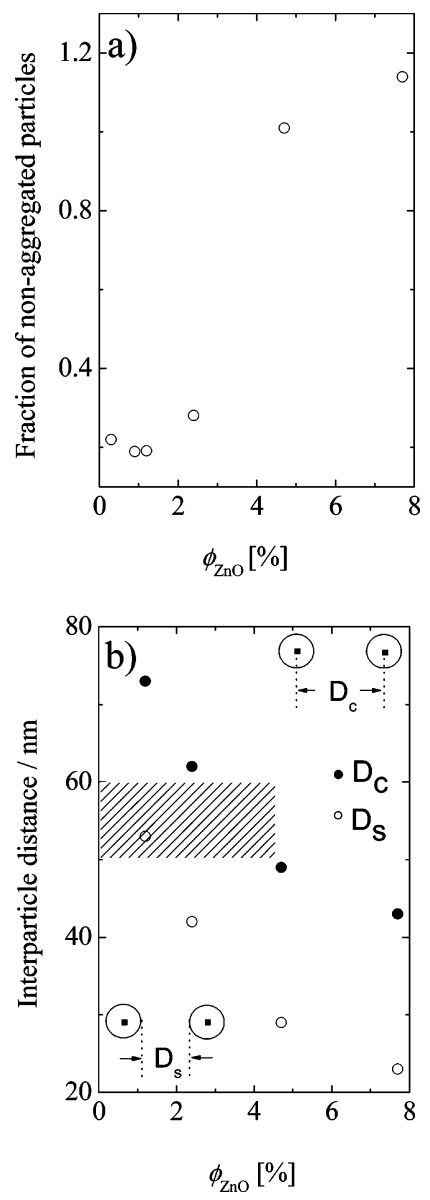

Figure 7. (a) Fraction of particles not involved in formation of particle clouds in PMMA. (b) Interparticle distance is calculated assuming a perfect dispersion of individual particles. Full and open symbols refer to center-to-center and surface-to-surface distances, respectively, for different particle contents (see text). The hatched area corresponds to a distance regime in which the excluded-volume effect and therefore clustering need to be expected.

large particles is tolerated in dilute systems. ${ }^{59}$ At higher concentrations, they cannot be ignored, and a significant deviation from Beer's law occurs as discussed above. We believe that the extent of deviation gives important information about the level of aggregation of the individual particles. Comparison of absorption data points with the values expected if Beer's law would hold provides the fraction of the particles which are not involved in formation of particle clouds. Figure 7a shows the volume fractions of these particles as depending on the overall concentration. This evaluation assumes that the total number of particles can be divided into two fractions: those which form clouds are mainly responsible for optical loss by scattering, and those which remain distributed about the remaining volume of the filler as individual species and, therefore, contribute mainly to loss by absorption.

The structure of the polymer-particle interface, i.e., the mutual arrangement of chains and filler particles, is of fundamental importance when discussing miscibility of filler particles within a polymer matrix. It has been recently proposed that a thermodynamically stable dispersion of nanoparticles without agglomeration or phase separation should be achieved when the radius of gyration, $R_{\mathrm{g}}$, of the polymer host is larger than the radius of the nanoparticles. ${ }^{60}$ Hooper and Schweizer ${ }^{16}$ suggested that $R_{\mathrm{g}}$ is not the key variable, but rather the ratio of particle diameter to monomer $(D / d)$ is crucial magnitude taking dense polymer-particle mixtures as a model system. This size ratio is relevant for the magnitude of particle-particle attraction. The 

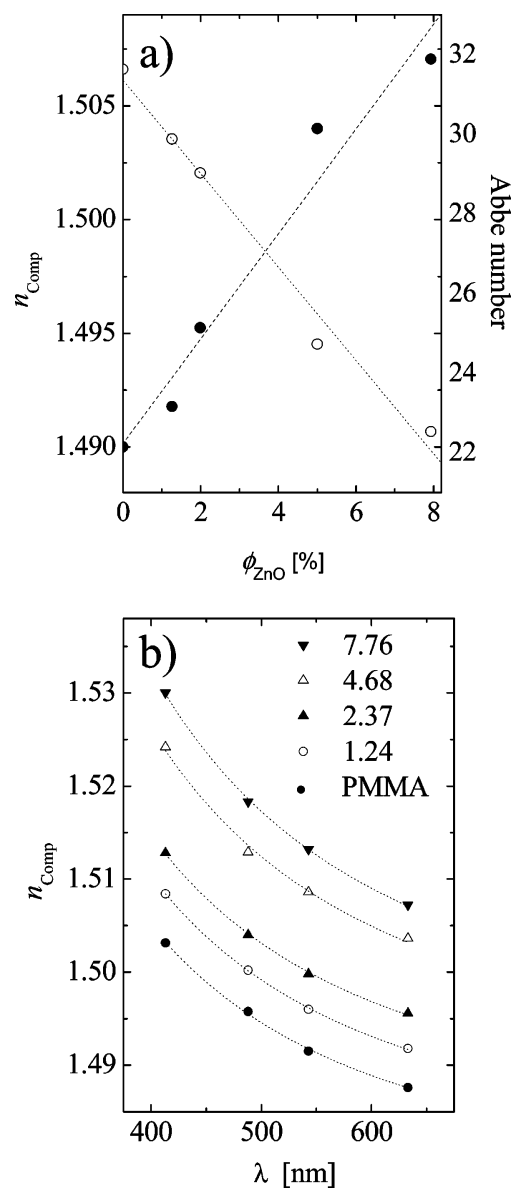

Figure 8. (a) Refractive index (full symbols) and the Abbe number (open symbols) at $633 \mathrm{~nm}$ as a function of the zincite content of the composite films. (b) Refractive indices of the composites having various volume fractions of $\mathrm{ZnO}$ measured at four wavelengths in the visible (symbols) and the corresponding fits by Cauchy's dispersion eq 4 (lines).

potential describing the mean force between the particles scales linearly with $D / d$. Depending on the strength of interparticle attractions, the latter authors proposed a number of qualitatively different behaviors for hard spheres such as contact aggregation, bridging, steric stabilization, and telebridging. Following the suggestions of Hooper and Schweizer, ${ }^{16}$ we are allowed to speculate that in our particular system, where the low interfacial energy and low interparticle attraction are present, steric stabilization is the dominating feature. In this type of organization the particles possess a distinct, thermodynamically stable noninterpenetrating "bound layer" of 2-3 monomers thickness. Here the term "monomer" refers to the quantity which enters the statistical description of polymer-polymer interactions; its spatial extinction $d$ may be assumed to be order of $1 \mathrm{~nm}$ in the case of PMMA. This surface layer of monomers acts as a cushion between the particles. This behavior corresponds to repulsive interactions between the particles and hence homogeneous dispersion of particles in the polymer matrix, especially at low concentrations. Since each particle has a thermodynamically stable "bound layer", the effective diameter and volume fraction of particles in the polymer matrix are larger than their "naked diameter" measured by DLS. Figure 7b gives the calculated interparticle distances (surface-to-surface and centerto-center) if the particles were well-dispersed free of aggregates. Increasing the particle content naturally decreases the separation of the particles. The interparticle center-to-center distance $\left(D_{\mathrm{c}}\right)$ scales with $r\left[\left(1 / \phi_{\mathrm{ZnO}}{ }^{1 / 3}\right)\right]$, but we may also consider the surfaceto-surface distance $D_{\mathrm{s}}=D_{c}-2 r$. The limiting value of $D_{\mathrm{s}}$ is
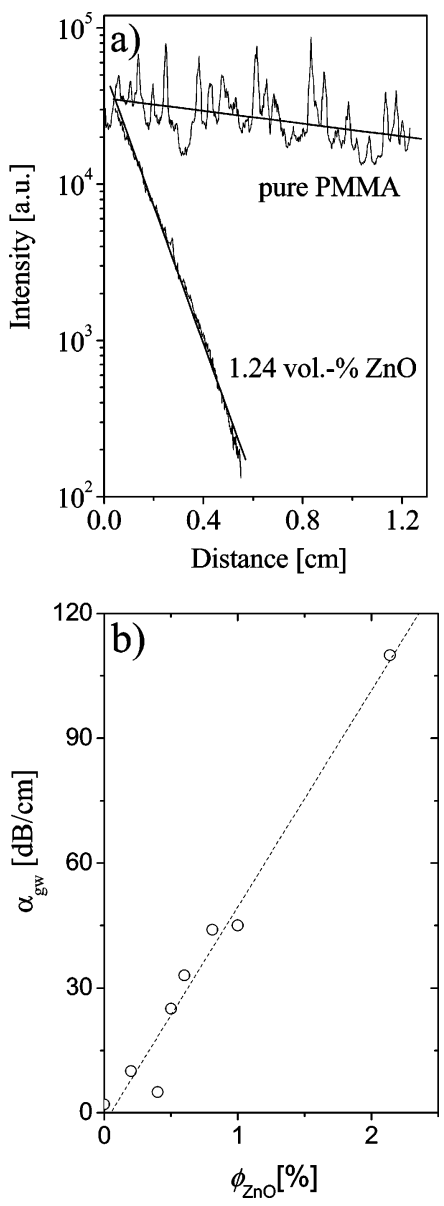

Figure 9. (a) Intensity of the light scattered from $\mathrm{TE}_{0}$ modes of waveguides of pure PMMA and PMMA/ZnO composites vs distance from the coupling prism at $\lambda=633 \mathrm{~nm}$. The slopes of the lines represent the loss coefficients $\alpha_{\mathrm{gw}}$. The fluctuation of transmitted light intensity in PMMA is mainly due to extrinsic losses such as impurities and dust. This fluctuation is barely observed for the composite films because of strong scattering of the $\mathrm{ZnO}$ particles. (b) Waveguide propagation loss coefficients $\alpha_{\mathrm{gw}}$ of $2 \mu \mathrm{m}$ thick PMMA/ZnO composite films vs the $\mathrm{ZnO}$ content measured at laser wavelength of $633 \mathrm{~nm}$.

$22 \mathrm{~nm}$ in the present case, that is, the first diameter of the "naked" particles. As Figure 7b shows, this value is reached at a $\phi_{\mathrm{ZnO}}$ of about 7.8 vol \%. However, we assumed that each particle is solvated by a shell of monomer units of a thickness of on average 3 layers thick $(3 \mathrm{~nm}) .{ }^{16}$ We need to consider an effective interparticle distance including the solvate shell. This results in a limiting value of $D_{\text {eff }}$ of $28 \mathrm{~nm}$. Moreover, excludedvolume effects need to be considered. For the simplest case of spheres the smallest center-to-center distance between the particles $\mathrm{A}$ and $\mathrm{B}$ should not be smaller than $2 D_{\text {eff }}$; otherwise, a third particle cannot be accommodated between them. This leads to a minimum center-to-center distance of about $56 \mathrm{~nm}$, at which a homogeneous dispersion of individual particles may be acceptable in the present case (see the hatched area in Figure $7 b)$. In other words, increasing the particle concentration beyond ca. $2 \mathrm{vol} \%$ must lead to the formation of aggregated or "clouds" as is experimentally observed. Thus, the segregation of the particles cannot be avoided upon polymerization even if the particles are homogeneously dispersed in the monomer.

The waveguide prism coupling technique was used to determine the refractive indices of PMMA/ZnO samples of various compositions and of neat reference PMMA, and they were measured at four different wavelengths covering the visible region. It was found as expected that the increase of the content of $\mathrm{ZnO}$ particles leads to a significant increase of the refractive 


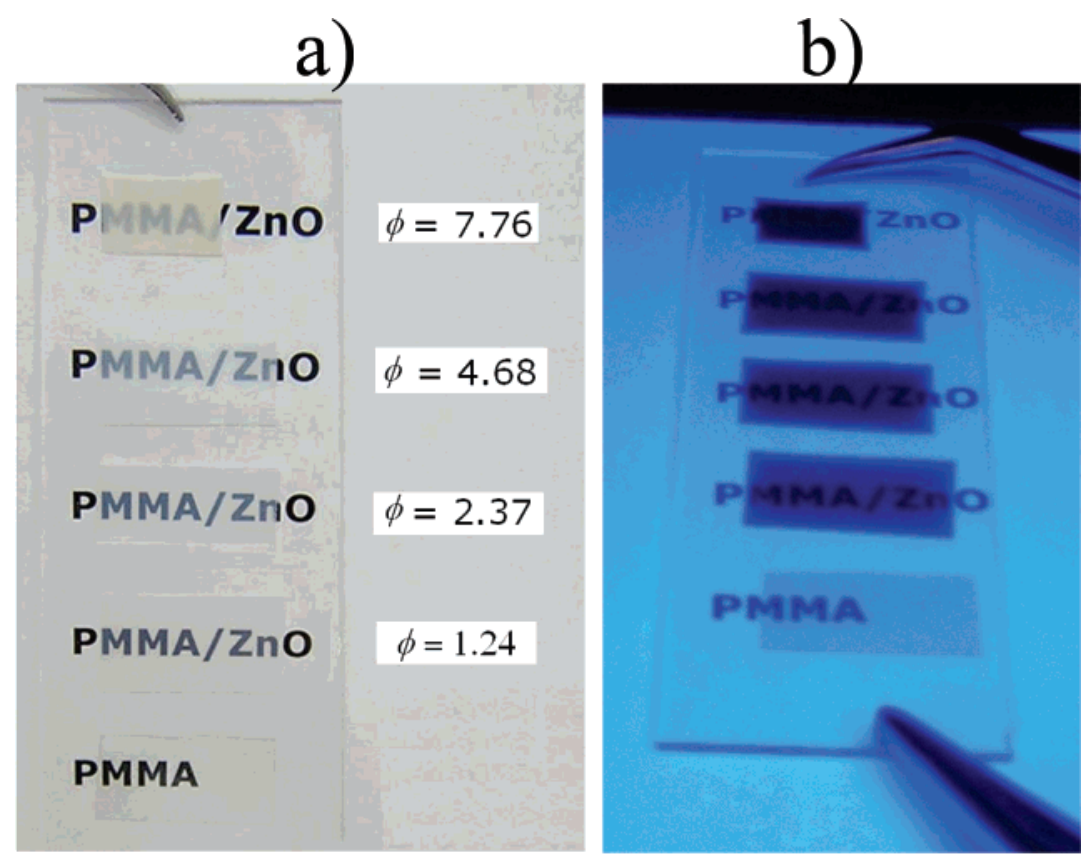

Figure 10. Photographs of the composite films having $120 \mu \mathrm{m}$ thickness under (a) daylight and (b) UV $\lambda=366 \mathrm{~nm}$. Percent volume fractions of $\mathrm{ZnO}$ are indicated in the images.

index of the composite. For example, while the refractive index of neat PMMA was measured to be 1.487 at $633 \mathrm{~nm}$, its value increases to 1.507 for the composite containing $7.76 \mathrm{vol} \%$ of $\mathrm{ZnO}$. An approximately linear relationship was found between the index of refraction and the volume fraction of particles (as shown in Figure 8a). Extrapolation of the linear fit to $100 \%$ $\mathrm{ZnO}$ content, however, results in a value of 1.73 , which is far less than that of the bulk $\mathrm{ZnO}{ }^{55}$ Similar results have been reported for different particle/polymer nanocomposite systems with about $10 \%$ negative deviation from the corresponding bulk material. ${ }^{61,62}$ We see two main reasons for such a discrepancy. The first one could be traced to the density difference between the inorganic core and the organic shell of the surface-modified $\mathrm{ZnO}$ nanoparticles. The organic shell contributes to the volume of the composite but has probably only a small contribution to the overall refractive index. Considering the core-shell structure as an object, the organic shell contributes a substantial fraction to the entire volume of the small particles although the thickness of the coating is limited by the size of the surfactant species. Therefore, core-shell structured $\mathrm{ZnO}-$ surfactant particles should exhibit a lower refractive index than bare $\mathrm{ZnO}$ particles. The second source of the deviation could be related to a smaller refractive index of the naked nanoparticles as compared to the bulk material due to quantum confinement.

The refractive index varies with wavelength, called dispersion. This variation is commonly quantified by the Abbe number as defined in the Experimental Section. It reflects the slope of the dispersion curve of a given material. Abbe numbers of the composite films are also shown in Figure 8a. The Abbe numbers decrease with $\mathrm{ZnO}$ content. This dispersion of the refractive index can be described by the phenomenological Cauchy relation: ${ }^{63}$

$$
n=A+\frac{B}{\lambda^{2}}+\frac{C}{\lambda^{4}}
$$

where $n$ is the refractive index, $\lambda$ is the wavelength, and $A, B$, and $C$ are constants. Figure $8 \mathrm{~b}$ presents both the measured refractive indices of the composite films and the corresponding fitting curves obtained by eq 4 . The fitting accounts reasonably
Table 2. Cauchy Coefficients of the Composite Films with Various $\mathrm{ZnO}$ Contents

\begin{tabular}{cclc}
\hline$\phi_{\mathrm{ZnO}}(\%)$ & $A$ & \multicolumn{1}{c}{$B\left(\mu \mathrm{m}^{2}\right)$} & $C\left(\mu \mathrm{m}^{4}\right)$ \\
\hline 0 & 1.476 & 4643 & $-5 \times 10^{-9}$ \\
1.24 & 1.479 & 4963 & $-5 \times 10^{-9}$ \\
2.37 & 1.482 & 5139 & $-5 \times 10^{-9}$ \\
4.68 & 1.487 & 6118 & $-5 \times 10^{-9}$ \\
7.76 & 1.490 & 6758 & $-5 \times 10^{-9}$ \\
$100^{64}$ & 1.940 & $-1.97 \times 10^{-5}$ & $7 \times 10^{-3}$
\end{tabular}

with the experimental results. The Cauchy coefficients of all PMMA/ZnO composites studied are summarized in Table 2. The values of the $A$ and $B$ increase with the increase of the particles content while the parameter $C$ stays constant for all composites. For comparison, the dispersion coefficients of bulk $\mathrm{ZnO}$ crystals as reported by Bond et al. ${ }^{64}$ are also shown in Table 2 .

In addition to the optical transmission $T$, the waveguide propagation loss is an important parameter which serves as quantitative measure for the optical quality of thin films. Highquality slab waveguides for applications in optoelectronics should have the smallest possible total waveguide propagation loss coefficient $\alpha_{\mathrm{gw}}$, which is defined as the sum of the intrinsic absorption coefficient $\alpha_{\text {abs }}$ and the scattering losses. Furthermore, because of the long propagation distance (typically 5-20 mm), the waveguide loss measurements are much more sensitive than transmission studies when optical losses in thin films need to be evaluated. As our composite films do not absorb in the visible and seem to show only a limited scattering loss (see Figure $6 a)$, we have measured $\alpha_{\mathrm{gw}}$ of slab waveguides made from the same composites with different contents of $\mathrm{ZnO}$ using the following procedure: A propagation mode in the waveguide was excited by prism coupling. The scattered light of the waveguide mode was imaged on a diode array (see the Experimental Section). Typical dependencies of the scattered light intensity as a function of the distance from the prism for the $\mathrm{TE}_{0}$ mode at the wavelength $633 \mathrm{~nm}$ are shown in Figure 9a. It indicates that the decrease of scattered light intensity with propagation distance is relatively small in the case of pure PMMA and significantly larger for the composite with only 1.24 vol $\% \mathrm{ZnO}$. As the intensity of the light scattered out of the 
film is proportional to the local guided wave intensity, the waveguide loss coefficient $\alpha_{\mathrm{gw}}$ is determined by the slope of the linear fit of the data, as shown in the example of Figure 9a.

We used this procedure to evaluate $\alpha_{\mathrm{gw}}$ of several waveguides made from pure PMMA and from composites with $\mathrm{ZnO}$ concentration in the range from 0.10 to $1.24 \mathrm{vol} \%$. The results are summarized in Figure 9b. As can be seen, the loss of the studied waveguides increased linearly from $2.5 \mathrm{~dB} \mathrm{~cm}^{-1}$ (PMMA) to $110 \mathrm{~dB} \mathrm{~cm}^{-1}$ with increasing $\mathrm{ZnO}$ content. The best fit to the data has a slope of $m=52 \mathrm{~dB} \mathrm{~cm}^{-1}$ vol $\%^{-1}$. Obviously, the strong increase of the waveguide loss in the composite material is related to the significant scattering power of the inorganic particles even at relatively low concentrations of the $\mathrm{ZnO}$. This is probably related not only to the high density of scattering centers (particles) and short scattering mean free path (short interparticle distance) but also to the increase of effective particle size due to aggregation in form of "clouds". This result corroborates the discussion centered on the data in Figure 7b. We were not able to measure $\alpha_{\mathrm{gw}}$ at $\mathrm{ZnO}$ concentrations higher than $1.24 \mathrm{vol} \%$ because the propagation length of the waveguide mode was too short for quantitative evaluations. While the above-reported loss values of the composite materials are significantly higher than typically required for integrated optics applications, i.e., $\sim 1 \mathrm{~dB} / \mathrm{cm},{ }^{65}$ they are still very suitable for applications like thin optical coatings where losses as at this level are acceptable.

As expressed in eq 3, transmission of such particle/polymer optical composites depends mainly on the particle content and the thickness of the material examined. The scattering intensity that causes the transmission loss is proportional to the scattering volume, i.e., thickness. So far, we focused on the spin-coated composite films having $2 \mu \mathrm{m}$ thickness. Thicker films of $\sim 120$ $\mu \mathrm{m}$ were prepared by film-casting from toluene solution. Figure 10 shows photographs of these films under both daylight and UV (at $366 \mathrm{~nm}$ ) at various contents of $\mathrm{ZnO}$ nanoparticles incorporated into the PMMA. In order to observe the effects of particles on the optical behavior of the composites, unfilled PMMA is given in both cases as a reference. A significant haziness is observed as the particle content increases above 1.25 vol \%. However, an acceptable transmission is still achieved even if the particle content is increased to $4.95 \mathrm{vol} \%$. While the particles appear as colorless under daylight, the presence of $\mathrm{ZnO}$ darkens the films in UV light because of strong UV absorption even at the lowest $\mathrm{ZnO}$ content, as can be seen in Figure 10b.

\section{Conclusion}

Composites of PMMA and hydrophobic zincite nanoparticles were prepared by polymerization of dispersions of the particles in the monomer (MMA). The $\mathrm{ZnO}$ particles were fabricated separately (ex situ) from $\mathrm{Zn}(\mathrm{OAc})_{2} \cdot 2 \mathrm{H}_{2} \mathrm{O}$ as the source. The particles have crystalline core of $\mathrm{ZnO}$ and are surface-modified with a strongly bound tert-butylphosphonate shell which allows to redisperse the particles into the monomer. The size distribution of the particles in MMA exhibited a maximum at $22 \mathrm{~nm}$ with a cutoff around $80 \mathrm{~nm}$. As a consequence of polymerization of MMA, the individual particles undergo aggregation to form domains of loosely associated particles. These domains or clouds start to overlap at higher concentrations of filler. However, the aggregates of the particles are uniformly distributed throughout the matrix without formation of micron or submicron scale $\mathrm{ZnO}$ domains. It is argued that the formation of these aggregates is a consequence of excluded-volume effects which have their origin mainly in constraints that arise from salvation of the particles by polymer chains. These findings shed light on principal problems which will occur when one tries to modify bulk polymers of nanosized particles in order to modify the optical properties of the polymers. The optical properties of PMMA respond to the incorporation of $\mathrm{ZnO}$ particles. Strong UV absorption, light transmittance in visible, and moderately high refractive index can make these composites very efficient in coatings of optical and electronic devices. We believe that the synthetic pathway for the preparation of optical composites is compatible with the industrial production of PMMA and is amenable to scaling up under conditions commonly found in industry.

\section{References and Notes}

(1) Huynh, W. U.; Dittmer, J. J.; Alivisatos, A. P. Science 2002, 295, $2425-2427$

(2) Arango, A. C.; Johnson, L. R.; Bliznyuk, V. N.; Schlesinger, Z.; Carter, S. A.; Horhold, H. H. Adv. Mater. 2000, 12, 1689-1692.

(3) Beecroft, L. L.; Ober, C. K. Chem. Mater. 1997, 9, 1302-1317.

(4) Yang, H. L.; Quan, R.; Zhang, G. H.; Chow, Y. T.; Chan, H. P.; Chu, P. L. Opt. Laser Technol. 2005, 37, 259-264.

(5) Chan, C. K.; Peng, S. L.; Chu, I. M.; Ni, S. C. Polymer 2001, 42 , 4189-4196.

(6) Yuwono, A. H.; Xue, J. M.; Wang, J.; Elim, H. I.; Ji, W.; Li, Y.; White, T. J. J. Mater. Chem. 2003, 13, 1475-1479.

(7) Lee, L. H.; Chen, W. C. Chem. Mater. 2001, 13, 1137-1142.

(8) Mataki, H.; Yamaki, S.; Fukui, T. Jpn. J. Appl. Phys., Part 12004 43, 5819-5823.

(9) Schulz, H.; Madler, L.; Pratsinis, S. E.; Burtscher, P.; Moszner, N. Adv. Funct. Mater. 2005, 15, 830-837.

(10) Bohm, J.; Hausselt, J.; Henzi, P.; Litfin, K.; Hanemann, T. Adv. Eng. Mater. 2004, 6, 52-57.

(11) Balazs, A. C.; Emrick, T.; Russell, T. P. Science 2006, 314, 11071110.

(12) Jain, A.; Wiesner, U. Macromolecules 2004, 37, 5665-5670.

(13) Thompson, R. B.; Ginzburg, V. V.; Matsen, M. W.; Balazs, A. C. Macromolecules 2002, 35, 1060-1071.

(14) Lin, Y.; Boker, A.; He, J. B.; Sill, K.; Xiang, H. Q.; Abetz, C.; Li, X F.; Wang, J.; Emrick, T.; Long, S.; Wang, Q.; Balazs, A.; Russell, T. P. Nature (London) 2005, 434, 55-59.

(15) Bockstaller, M. R.; Lapetnikov, Y.; Margel, S.; Thomas, E. L. J. Am. Chem. Soc. 2003, 125, 5276-5277.

(16) Hooper, J. B.; Schweizer, K. S. Macromolecules 2005, 38, 88588869.

(17) Buxton, G. A.; Balazs, A. C. J. Chem. Phys. 2002, 117, 7649-7658.

(18) Hung, C. H.; Whang, W. T. J. Mater. Chem. 2005, 15, 267-274.

(19) Caseri, W. Macromol. Rapid Commun. 2000, 21, 705-722.

(20) Kickelbick, G. Prog. Polym. Sci. 2003, 28, 83-114.

(21) KyprianidouLeodidou, T.; Margraf, P.; Caseri, W.; Suter, U. W.; Walther, P. Polym. Adv. Technol. 1997, 8, 505-512.

(22) Mulligan, R. F.; Iliadis, A. A.; Kofinas, P. J. Appl. Polym. Sci. 2003, 89, 1058-1061.

(23) Mikrajuddin Lenggoro, I. W.; Okuyama, K.; Shi, F. G. J. Electrochem. Soc. 2002, 149, H107-H112.

(24) Bockstaller, M. R.; Mickiewicz, R. A.; Thomas, E. L. Adv. Mater. 2005, 17, 1331-1349.

(25) De Paul, S. M.; Zwanziger, J. W.; Ulrich, R.; Wiesner, U.; Spiess, H. W. J. Am. Chem. Soc. 1999, 121, 5727-5736.

(26) Demir, M. M.; Gulgun, M. A.; Menceloglu, Y. Z.; Erman, B.; Abramchuk, S. S.; Makhaeva, E. E.; Khokhlov, A. R.; Matveeva, V. G.; Sulman, M. G. Macromolecules 2004, 37, 1787-1792.

(27) Wen, J. Y.; Wilkes, G. L. Chem. Mater. 1996, 8, 1667-1681.

(28) Su, W. F.; Lee, J. F.; Chen, M. Y.; Ho, R. M. J. Mater. Res. 2004 19, 2343-2348.

(29) Palkovits, R.; Althues, H.; Rumplecker, A.; Tesche, B.; Dreier, A.; Holle, U.; Fink, G.; Cheng, C. H.; Shantz, D. F.; Kaskel, S. Langmuir 2005, 21, 6048-6053.

(30) Hingorani, S.; Pillai, V.; Kumar, P.; Multani, M. S.; Shah, D. O. Mater. Res. Bull. 1993, 28, 1303-1310.

(31) Tang, E. J.; Cheng, G. X.; Pang, X. S.; Ma, X. L.; Xing, F. B. Colloid Polym. Sci. 2006, 284, 422-428.

(32) Advincula, R. C. J. Dispersion Sci. Technol. 2003, 24, 343-361.

(33) Masala, O.; Seshadri, R. Annu. Rev. Mater. Res. 2004, 34, 41-81.

(34) Ulrich, R.; Torge, R. Appl. Opt. 1973, 12, 2901-2908.

(35) Farn, M. W.; Veldkamp, W. B. In Handbook of Optics, II ed.; Bass, M., Ed.; McGraw-Hill: Belfast, 1995; Vol. 2, p 8.6.

(36) Mathy, A.; Simmrock, H. U.; Bubeck, C. J. Phys. D: Appl. Phys. 1991, 24, 1003-1008 
(37) Demir, M. M.; Espi-Munoz, R.; Lieberwirth, L.; Wegner, G. J. Mater. Chem. 2006, 16, 2940-2947.

(38) Choy, J. H.; Kwon, Y. M.; Han, K. S.; Song, S. W.; Chang, S. H. Mater. Lett. 1998, 34, 356-363.

(39) Goodwin, J. Colloids and Interfaces with Surfactants and Polymers, 1st ed.; John Wiley \& Sons Ltd.: London, 2004.

(40) Cozzoli, P. D.; Curri, M. L.; Agostiano, A.; Leo, G.; Lomascolo, M. J. Phys. Chem. B 2003, 107, 4756-4762.

(41) Mehring, M.; Schurmann, M.; Ludwig, R. Chem.-Eur. J. 2003, 9, 838-849.

(42) Pawsey, S.; McCormick, M.; De, Paul, S.; Graf, R.; Lee, Y. S.; Reven, L.; Spiess, H. W. J. Am. Chem. Soc. 2003, 125, 4174-4184.

(43) Pawsey, S.; Yach, K.; Reven, L. Langmuir 2002, 18, 5205-5212.

(44) Guerrero, G.; Mutin, P. H.; Vioux, A. Chem. Mater. 2001, 13, 43674373.

(45) Carriere, D.; Moreau, M.; Barboux, P.; Boilot, J. P.; Spalla, O. Langmuir 2004, 20, 3449-3455.

(46) McCafferty, E.; Wightman, J. P. Surf. Interface Anal. 1998, 26, 549564.

(47) Sahoo, Y.; Pizem, H.; Fried, T.; Golodnitsky, D.; Burstein, L.; Sukenik, C. N.; Markovich, G. Langmuir 2001, 17, 7907-7911.

(48) Randon, J.; Blanc, P.; Paterson, R. J. Membr. Sci. 1995, 98, 119129.

(49) Klug, H. P.; Alexander, L. E. X-Ray Diffraction Procedures for Polycrystalline and Amorphous Materials, 2nd ed.; John Wiley \& Sons: New York, 1974.

(50) Demir, M. M.; Memesa, M.; Castignolles, P.; Wegner, G. Macromol. Rapid Commun. 2006, 27, 763-770.

(51) Demir, M. M.; Menceloglu, Y. Z.; Erman, B. Mater. Chem. Phys. 2006, 207, 1515-1524.
(52) Novak, B. M. Adv. Mater. 1993, 5, 422-433.

(53) Lide, D. R. CRC Handbook of Chemistry and Physics; CRC Press: Boca Raton, FL, 1995.

(54) Wunderlich, W. In Polymer Handbook; Brandrup, J., Immergut, E. H., Grulke, E. A., Eds.; John Wiley and Sons: New York, 1999; p $\mathrm{V} / 87$.

(55) Ozgur, U.; Alivov, Y. I.; Liu, C.; Teke, A.; Reshchikov, M. A.; Dogan, S.; Avrutin, V.; Cho, S. J.; Morkoc, H. J. Appl. Phys. 2005, 98.

(56) Penzkofer, A.; Drotleff, E.; Holzer, W. Opt. Commun. 1998, 158, $221-$ 230.

(57) Muth, J. F.; Kolbas, R. M.; Sharma, A. K.; Oktyabrsky, S.; Narayan, J. J. Appl. Phys. 1999, 85, 7884-7887.

(58) Bohren, C. D.; Huffman, D. R. Absorption and Scattering of Light by Small Particles; John Wiley \& Sons: New York, 1983.

(59) Jones, A. R. Prog. Energy Combust. Sci. 1999, 25, 1-53.

(60) Mackay, M. E.; Tuteja, A.; Duxbury, P. M.; Hawker, C. J.; Van, Horn, B.; Guan, Z. B.; Chen, G. H.; Krishnan, R. S. Science 2006, 311 , $1740-1743$

(61) Kyprianidouleodidou, T.; Caseri, W.; Suter, U. W. J. Phys. Chem. 1994, 98, 8992-8997.

(62) Nussbaumer, R. J.; Caseri, W. R.; Smith, P.; Tervoort, T. Macromol Mater. Eng. 2003, 288, 44-49.

(63) Tropf, W. J.; Thomas, M. E.; Harris, T. J. In Handbook of Optics, II ed.; Bass, M., Ed.; McGraw-Hill: Belfast, 1995; Vol. 2, p 33.26.

(64) Bond, W. L. J. Appl. Phys. 1965, 36, 1674-1677.

(65) Ma, H.; Jen, A. K. Y.; Dalton, L. R. Adv. Mater. 2002, 14, 1339-1365.

MA062184T 